\title{
Variabilidade genética de caracteres forrageiros em Paspalum
}

\author{
Emerson André Pereira(1), Thiago Barros ${ }^{(1)}$, Gabriela Kessler Volkmann ${ }^{(1)}$, Gabriel Koltermann Battisti(2), \\ José Antonio Gonzalez da Silva( ${ }^{(2)}$, Carine Simioni( ${ }^{(1)}$ e Miguel Dall'Agnol(1)
}

\begin{abstract}
(1) Universidade Federal do Rio Grande do Sul, Faculdade de Agronomia, Departamento de Plantas Forrageiras e Agrometeorologia, Avenida Bento Gonçalves, no7.712, CEP 91501-970 Porto Alegre, RS. E-mail: emersonpijui@yahoo.com.br, thiagopoabr@hotmail.com, gabivolkmann@hotmail.com, carine.simioni@ufrgs.br, migueld@ufrgs.br(2)Universidade Regional do Noroeste do Estado do Rio Grande do Sul, Departamento de Estudos Agrários, Rua do Comércio, no 3.000, CEP 98700-000 ljuí, RS. E-mail: gabrielkbattisti@bol.com, jagsfaem@yahoo.com.br
\end{abstract}

Resumo - O objetivo deste trabalho foi determinar a variabilidade genética e a expressão de caracteres de interesse forrageiro em espécies de Paspalum. Os experimentos foram conduzidos em diferentes locais e anos de cultivo, em delineamento de blocos ao acaso, com três repetições. Foram avaliados cinco acessos de $P$. nicorae e dois de $P$. guenoarum, além da cultivar Pensacola ( $P$. notatum), utilizada como testemunha. Foram quantificados os seguintes caracteres: relação folha/colmo, índice de colheita e massa de matéria seca total, de folhas e de colmo. Tanto os efeitos principais (genótipos, anos e locais de cultivo) quanto a interação entre os fatores tiveram influência significativa sobre os caracteres avaliados. Os acessos avaliados apresentam variabilidade genética em caracteres de interesse forrageiro, bem como desempenho variável de acordo com o local e o ano de cultivo. A produção de matéria seca total e de folhas são os caracteres que mais contribuem para a detecção da variabilidade genética observada, independentemente do ano de avaliação.

Termos para indexação: apomixia, espécie nativa, interação genótipo x ambiente, seleção.

\section{Genetic variability of forage traits in Paspalum}

\begin{abstract}
The objective of this work was to determine the genetic variability and the expression of traits of interest for forage production in species of Paspalum. The experiments were carried out in different locations and crop years in randomized block design with three replicates. Five accessions of $P$. nicorae and two of $P$. guenoarum were evaluated, besides the cultivar Pensacola ( $P$. notatum), used as a control. The following traits were determined: leaf/stalk ratio; harvest index; and total, leaf, and stalk dray matter. Both main effects (genotype, years, and locations) and the interactions among factors had significant influence on the evaluated traits. The evaluated accessions showed genetic variability in traits of interest for forage production, and their performance varied according to the location and year of cultivation. Total and leaf dry matter production are the traits that most contribute to the detection of the observed genetic variability, regardless of the year of evaluation.
\end{abstract}

Index terms: apomixis, native species, genotype $\mathrm{x}$ environment interaction, selection.

\section{Introdução}

No Rio Grande do Sul, há espécies forrageiras nativas de vários gêneros, com bom valor nutritivo, que constituem a base da exploração pecuária na região, pois, embora as forrageiras exóticas tenham elevado potencial de produção, muitas vezes elas não se adaptam às condições edafoclimáticas locais.

Entre as espécies nativas encontradas no Sul do Brasil, destacam-se as do gênero Paspalum, que apresentam elevado potencial forrageiro e são adaptadas a diversos ecossistemas brasileiros (Batista \& Godoy, 2000). Para Strapasson et al. (2000), o fato dessas espécies comporem o ecossistema nativo acarreta menor risco de desequilíbrio biológico pela exploração pecuária. Em razão da grande variabilidade intra e interespecífica existente no gênero Paspalum, há grande potencial para sua exploração em programas de melhoramento genético (Batista \& Godoy 2000; Reis et al., 2010; Pereira et al., 2011).

A maioria das espécies do gênero Paspalum tem como modo de reprodução a apomixia, o que implica populações geneticamente homogêneas (Quarín \& Norrmann 1990). A apomixia dificulta a recombinação genética, assim como a proteção de cultivares pelas normas legais vigentes. O desenvolvimento dessas espécies em ambientes divergentes também pode ser dificultado (baixa estabilidade fenotípica), pois não

Pesq. agropec. bras., Brasília, v.47, n.10, p.1533-1540, out. 2012 
há troca de alelos favoráveis à adaptação. Este fato aumenta os riscos de perda de genótipos por fatores abióticos e bióticos (Adamowski et al., 2005). Porém, a utilização de hibridizações, quando um dos genitores apresenta reprodução sexuada, pode gerar variabilidade e possibilitar a seleção de progênies elite, com fixação imediata dos caracteres de interesse em razão da apomixia (Rodrigues et al., 2003; Acuña et al., 2009).

A descoberta de plantas diploides sexuais em populações naturais de $P$. plicatulum possibilitou a obtenção de novos genótipos a partir de cruzamentos com espécies compatíveis (Sartor et al., 2009). Essa descoberta teve forte impacto sobre programas de melhoramento das plantas forrageiras do grupo Plicatula, antes impossibilitados pela apomixia. Aguilera et al. (2011) hibridizaram artificialmente um genótipo de $P$. plicatulum, denominado " $4 \mathrm{c}-4 \mathrm{x}$ ” (genitor feminino), com a cultivar apomítica conhecida como Rojas (P. guenoarum, genitor masculino), e obtiveram 23 híbridos interespecíficos viáveis.

O conhecimento da expressão de caracteres de interesse forrageiro e da variabilidade genética existente entre genótipos de diferentes espécies do gênero Paspalum pode contribuir para a identificação de plantas superiores quanto à produção de forragem, e para o direcionamento de cruzamentos com genitor sexual, com vistas à seleção de recombinantes desejáveis.

O objetivo deste trabalho foi determinar a variabilidade genética e a expressão de caracteres de interesse forrageiro de acessos de espécies do gênero Paspalum, em diferentes locais e anos de cultivo.

\section{Material e Métodos}

O experimento foi realizado nos anos agrícolas de 2009/2010 e 2010/2011, nos municípios de Eldorado do Sul e Augusto Pestana, RS. A área experimental em Eldorado do Sul está localizada na região da Depressão Central ( $30^{\circ} 06^{\prime} 02^{\prime \prime} \mathrm{S}$ e $51^{\circ} 41^{\prime} 27^{\prime \prime} \mathrm{W}$, a $34 \mathrm{~m}$ de altitude). O solo é classificado como Argissolo Vermelho distrófico típico, e o clima, segundo a classificação de Köppen, é do tipo $\mathrm{Cfa}$, subtropical úmido, com verão quente. A temperatura média anual é de $19,3^{\circ} \mathrm{C}$, com média máxima de $24,6^{\circ} \mathrm{C}$, em janeiro, e média mínima de $13,8^{\circ} \mathrm{C}$, em junho. A precipitação média anual está em torno de $1.400 \mathrm{~mm}$. Durante o período de avaliação, choveu $1.692 \mathrm{~mm}$ no primeiro ano e 807 no segundo, e a temperatura média esteve em torno de $19^{\circ} \mathrm{C}$. Em Augusto Pestana, a área experimental está situada na região Noroeste do estado $\left(28^{\circ} 25^{\prime} 58^{\prime \prime} \mathrm{S}\right.$ e $54^{\circ} 00^{\prime} 24^{\prime \prime} \mathrm{W}$, a $290 \mathrm{~m}$ de altitude). O solo é classificado como Latossolo Vermelho distroférrico típico, e o clima é do tipo Cfa, conforme a classificação de Köppen. A precipitação média é de $1.600 \mathrm{~mm}$, e a temperatura média é de $21,8^{\circ} \mathrm{C}$. Janeiro e fevereiro são os meses mais quentes do ano, com temperatura superior a $22^{\circ} \mathrm{C}$, enquanto junho e julho são os meses mais frios, com temperatura média entre 3 e $18^{\circ} \mathrm{C}$. Durante a condução do experimento, choveu $1.711 \mathrm{~mm}$ no primeiro ano e 1.200 no segundo, com temperatura média de $20^{\circ} \mathrm{C}$.

Foram realizadas análises do solo no período que antecedeu a implantação do experimento, em ambos os locais. As amostras foram retiradas da camada de 0 a $20 \mathrm{~cm}$. Em Eldorado do Sul, o solo apresentou as seguintes características: $210 \mathrm{~g} \mathrm{~kg}^{-1}$ de argila; $\mathrm{pH}$, 5,4; índice SMP de 6,3; $14 \mathrm{~g} \mathrm{~kg}^{-1}$ de matéria orgânica; $3,1 \mathrm{cmol}_{\mathrm{c}} \mathrm{dm}^{-3} \mathrm{de} \mathrm{H}+\mathrm{Al} ; 6,7 \mathrm{mg} \mathrm{dm}^{-3}$ de P; $119 \mathrm{mg} \mathrm{dm}^{-3}$ de K; eAl, Ca, Mg e CTC de 0,1, 2,0, 0, 7, 6, $1 \mathrm{cmol}_{\mathrm{c}} \mathrm{dm}^{-3}$, respectivamente; com saturação por bases de $49 \%$ e por Al de 3,2\%. Em Augusto Pestana, o solo apresentou: $560 \mathrm{~g} \mathrm{~kg}^{-1}$ de argila; $\mathrm{pH}, 6,2$; índice SMP de 6,$1 ; 26 \mathrm{~g} \mathrm{~kg}^{-1}$ de matéria orgânica; $3,9 \mathrm{cmol}_{\mathrm{c}} \mathrm{dm}^{-3}$ de $\mathrm{H}+\mathrm{Al} ; 46,2 \mathrm{mg} \mathrm{dm}^{-3}$ de P; $215 \mathrm{mg} \mathrm{dm}^{-3}$ de $\mathrm{K}$; e $\mathrm{Al}, \mathrm{Ca}, \mathrm{Mg}$ e CTC de 0, 7,2, 2,2 e 9,9 $\mathrm{cmol}_{\mathrm{c}} \mathrm{dm}^{-3}$, respectivamente; com percentagem da saturação por bases de 71,9\%; e por $\mathrm{Al}$ de $0 \%$. As áreas receberam adubação de base na quantidade de 20-150-100 e 20-120-100 kg ha-1 de N-P-K, em Eldorado do Sul e Augusto Pestana, respectivamente. Foram realizadas adubações de cobertura de 180 e $130 \mathrm{~kg} \mathrm{ha}^{-1}$ de $\mathrm{N}$ em Eldorado do Sul e Augusto Pestana, respectivamente. As adubações de cobertura foram fracionadas em seis momentos. As aplicações foram realizadas após cada corte, conforme as indicações técnicas para gramíneas perenes de estação quente, seguindo as recomendações da Rede Oficial de Laboratórios de Análise de Solos (Manual de adubação e de calagem para os Estados do Rio Grande do Sul e Santa Catarina, 2004).

Os acessos utilizados foram obtidos de coleção pertencente ao Departamento de Plantas Forrageiras e Agrometeorologia, da Universidade Federal do Rio Grande do Sul, a partir de coletas realizadas em ecossistemas naturais do estado (Reis et al., 2010; Pereira et al., 2011). Os acessos de maior potencial forrageiro, determinado em estudo preliminar, foram 
selecionados para a realização do experimento: P. nicorae (acessos: 28B, 26A, 28C, 26D e 28E) e P. guenoarum (acessos: Azulão e Baio). Além disso, foi incluída, como testemunha, a cultivar Pensacola ( $P$. notatum), também proveniente das condições naturais do Cone Sul e que apresenta alta relação folha/ colmo e qualidade nutricional (Pereira et al., 2011).

Em cada local, utilizou-se o delineamento experimental de blocos ao acaso, com três repetições, em que as parcelas foram constituídas por seis linhas de 2,2 $\mathrm{m}$ de comprimento e $0,20 \mathrm{~m}$ de espaçamento. Em cada linha, as mudas foram transplantadas a cada $20 \mathrm{~cm}$, o que totalizou 60 plantas por parcela. Os transplantes ocorreram em 1 e 4 de dezembro de 2009, em Eldorado do Sul e Augusto Pestana, respectivamente.

As avaliações foram realizadas por meio de cortes, com a utilização de dois quadrados (gabarito) de 0,50x0,50 m, quando a maior parte dos acessos apresentava altura média das folhas de $35 \mathrm{~cm}$, tendo permanecido um resíduo de $10 \mathrm{~cm}$ do solo, exceto para a cultivar Pensacola, que foi cortada ao atingir altura média de $25 \mathrm{~cm}$, com resíduo de $5 \mathrm{~cm}$, manejo mais indicado para esta espécie (Sawasato, 2007; Pereira et al., 2011). Também foram utilizados dois quadrados, com $0,25 \times 0,25 \mathrm{~m}$, para a contagem do número de afilhos por área. Após os cortes, as amostras foram levadas ao laboratório para a separação morfológica de folhas, colmos, inflorescências e material morto, e secas em estufa de ar forçado, a $65^{\circ} \mathrm{C}$, até massa constante, para análise dos caracteres de interesse.

Os caracteres mensurados foram: massa de matéria seca total (MST, $\mathrm{kg} \mathrm{ha}^{-1}$ ), e folhas (MSF, $\mathrm{kg} \mathrm{ha}^{-1}$ ) e de colmo (MSC, $\mathrm{kg} \mathrm{ha}^{-1}$ ). Não foi considerado o material morto, por praticamente inexistir durante as avaliações. Com estas variáveis, foram calculados a relação folha/ colmo (RFC, MSF/MSC) e o índice de colheita (IC, $\mathrm{MSF} / \mathrm{MST}$ ). Foram realizados cinco cortes, no primeiro ano, e seis, no segundo, que foram somados dentro de cada ano e local, exceto para a cultivar Pensacola, para a qual foram realizados quatro e cinco cortes no primeiro e no segundo ano, respectivamente. O menor número de cortes da testemunha ocorreu em razão do pequeno crescimento das plantas, decorrente das baixas temperaturas no outono e no inverno. O número de afilhos (NAF) foi obtido por contagem, para obtenção da média produzida ao longo dos anos.
Os dados foram submetidos à análise de variância, tendo-se utilizado o teste $\mathrm{F}$, a $5 \%$ de probabilidade. As médias foram comparadas pelo teste de Scott-Knott, a $5 \%$ de probabilidade. Utilizou-se a análise multivariada, com emprego da distância generalizada de Mahalanobis $\left(\mathrm{D}^{2}\right)$, para estimar a variabilidade genética existente entre os acessos, em cada local e ano de cultivo, e o método de Tocher foi usado como técnica de agrupamento dos acessos. A magnitude de contribuição relativa das variáveis, para a variabilidade morfológica total observada nas diferentes condições estabelecidas, foi determinada pelo modelo de Singh (1981). Para estas determinações, foi utilizado o programa Genes (Cruz, 2007).

\section{Resultados e Discussão}

Tanto os efeitos principais (genótipo, anos e locais) como a interação entre os fatores (genótipo $\mathrm{x}$ ano, genótipo $\mathrm{x}$ local, ano $\mathrm{x}$ local e genótipo $\mathrm{x}$ ano $\mathrm{x}$ local) influenciaram significativamente os caracteres avaliados (Tabela 1). $\mathrm{O}$ ano de cultivo exerceu maior influência (quadrado médio mais elevado) sobre os caracteres MST, MSF, RFC e IC que os demais fatores. A MST e a MSF foram os caracteres mais efetivos na identificação de genótipos superiores, com aptidão forrageira; tendo sofrido forte influência da interação entre genótipos e ambiente de cultivo (Assis et al., 2008; Pereira et al., 2011).

No Município de Eldorado do Sul, os acessos Azulão e Baio de P. guenoarum apresentaram os melhores desempenhos na produção de MST, em comparação aos acessos das espécies $P$. nicorae e $P$. notatum, nos dois anos avaliados. Esses dados corroboram os obtidos por outros autores, que evidenciaram a alta produção desses acessos em avaliações com genótipos nativos, entre os quais a cultivar Pensacola (Sawasato, 2007; Pereira et al., 2011). Nesse município, a produção média de matéria seca total dos acessos, no segundo ano, foi maior do que a obtida no primeiro (Tabela 2). O melhor desempenho no segundo ano também foi observado no Município de Augusto Pestana. Este resultado pode ser atribuído ao processo de adaptação das mudas depois do transplante, que culminou com a maior capacidade de desenvolvimento e produção após a perenização. Em espécies de hábito rizomatoso ou estolonífero, o processo de adaptação da espécie ao ambiente ocorre no primeiro ano (Pereira et al., 2011). 
Tabela 1. Resumo da análise de variância de caracteres forrageiros de acessos do gênero Paspalum, em dois anos de cultivo, em Eldorado do Sul e Augusto Pestana, RS.

\begin{tabular}{|c|c|c|c|c|c|c|c|}
\hline \multirow[t]{2}{*}{ Fonte de variação } & \multirow[t]{2}{*}{ GL } & \multicolumn{6}{|c|}{ Quadrado médio } \\
\hline & & MST & MSF & MSC & RFC & IC (MSF/MST) & NAF por $\mathrm{m}^{2}$ \\
\hline (Bloco/Local)/Ano & 8 & 1.032 .399 & 579.238 & 87.565 & 0.59 & 0.012 & 43456 \\
\hline Genótipo (G) & 7 & $102.763 .861 *$ & $58.748 .634^{*}$ & $7063.331 *$ & $11.81 *$ & $0.04 *$ & $44.461^{*}$ \\
\hline $\operatorname{Anos}(A)$ & 1 & $145.679 .172 *$ & $212.656 .579 *$ & $325.306^{*}$ & $114^{*}$ & $1.42 *$ & $107.596^{*}$ \\
\hline Locais (L) & 1 & $2.675 .086^{*}$ & $761.562 *$ & $174.659^{*}$ & $0.05^{*}$ & $0.02 *$ & $1.560 .877^{*}$ \\
\hline $\mathrm{G} \times \mathrm{A}$ & 7 & 37.182.394* & $15.260 .521^{*}$ & $3.584 .427 *$ & $6.23^{*}$ & $0.03^{*}$ & $96.515^{*}$ \\
\hline Gx L & 7 & $5.754 .648^{*}$ & $3.421 .097^{*}$ & $546.681 *$ & $4.79 *$ & $0.02 *$ & $51.710^{*}$ \\
\hline$A \times L$ & 1 & 19.120.973* & $4.630^{*}$ & $11.691 .451^{*}$ & $16.06^{*}$ & $0.10^{*}$ & $246.303^{*}$ \\
\hline $\mathrm{G} \times \mathrm{A} \times \mathrm{L}$ & 7 & $3.316 .443 *$ & $2.159 .252 *$ & $1.573 .424 *$ & $11.70^{*}$ & $0.02 *$ & $58.776^{*}$ \\
\hline Erro & 56 & 1.014 .076 & 446.814 & 178.301 & 0.33 & 0.017 & 7.495 \\
\hline Total & 95 & - & - & - & - & - & - \\
\hline Média geral & & $6.401 \mathrm{~kg} \mathrm{ha}^{-1}$ & $4.248 \mathrm{~kg} \mathrm{ha}^{-1}$ & $1.705 \mathrm{~kg} \mathrm{ha}^{-1}$ & 2.95 & 0.64 & 571 \\
\hline CV $(\%)$ & & 15.73 & 15.73 & 24.75 & 19.42 & 6.41 & 15.15 \\
\hline
\end{tabular}

*Significativo a 5\% de probabilidade, pelo teste F. MST, massa de matéria seca total; MSF, massa de matéria seca de folha; MSC, massa de matéria seca de colmo; RFC, relação folha:colmo; IC, índice de colheita; NAF, número de afilhos.

Tabela 2. Produção de matéria seca total de folhas de colmo de acessos do gênero Paspalum, em dois anos de cultivo e em dois municípios do Rio Grande do Sul ${ }^{(1)}$.

\begin{tabular}{|c|c|c|c|c|}
\hline \multirow[t]{2}{*}{ Genótipo } & \multicolumn{2}{|c|}{ Eldorado do Sul } & \multicolumn{2}{|c|}{ Augusto Pestana } \\
\hline & $2009 / 2010$ & $2010 / 2011$ & $2009 / 2010$ & $2010 / 2011$ \\
\hline & \multicolumn{4}{|c|}{ Massa de matéria seca total $\left(\mathrm{kg} \mathrm{ha}^{-1}\right)$} \\
\hline P. guenoarum Azulão & $6.519 \mathrm{aB}-\mathrm{B}$ & $15.995 \mathrm{aA}-\mathrm{A}$ & $8.050 \mathrm{aA}-\mathrm{B}$ & $13.228 \mathrm{bB}-\mathrm{A}$ \\
\hline P. guenoarum Baio & $6.267 \mathrm{aB}-\mathrm{B}$ & $14.252 \mathrm{aB}-\mathrm{A}$ & $7.494 \mathrm{aA}-\mathrm{B}$ & $16.836 \mathrm{aA}-\mathrm{A}$ \\
\hline P. nicorae 26A & $3.894 \mathrm{bB}-\mathrm{A}$ & $4.941 \mathrm{bA}-\mathrm{A}$ & $5.525 \mathrm{bA}-\mathrm{A}$ & $5.758 \mathrm{cA}-\mathrm{A}$ \\
\hline P. nicorae 26D & $4.074 \mathrm{bA}-\mathrm{B}$ & $6.456 \mathrm{bA}-\mathrm{A}$ & $4.865 \mathrm{cA}-\mathrm{A}$ & $4.209 \mathrm{~dB}-\mathrm{A}$ \\
\hline P. nicorae $28 \mathrm{~B}$ & $4.529 \mathrm{bA}-\mathrm{B}$ & $8.130 \mathrm{bA}-\mathrm{A}$ & $5.384 \mathrm{bA}-\mathrm{A}$ & $5.000 \mathrm{cB}-\mathrm{A}$ \\
\hline P. nicorae $28 \mathrm{C}$ & $3.660 \mathrm{bA}-\mathrm{A}$ & $5.031 \mathrm{bA}-\mathrm{A}$ & $4.593 \mathrm{cA}-\mathrm{B}$ & $5.944 \mathrm{cA}-\mathrm{A}$ \\
\hline P. nicorae $28 \mathrm{E}$ & $3.554 \mathrm{bB}-\mathrm{A}$ & $2.722 \mathrm{bA}-\mathrm{A}$ & $5.904 \mathrm{bA}-\mathrm{A}$ & $4.796 \mathrm{cA}-\mathrm{A}$ \\
\hline P. notatum Pensacola & $3.953 \mathrm{bA}-\mathrm{A}$ & $5.772 \mathrm{bA}-\mathrm{A}$ & $4.443 \mathrm{cA}-\mathrm{A}$ & $3.057 \mathrm{~dB}-\mathrm{B}$ \\
\hline \multirow[t]{2}{*}{ Média geral } & 4.556 & 7.912 & 5.782 & 7.354 \\
\hline & \multicolumn{4}{|c|}{ Massa de matéria seca de folhas $\left(\mathrm{kg} \mathrm{ha}^{-1}\right)$} \\
\hline P. guenoarum Azulão & $5.520 \mathrm{aA}-\mathrm{B}$ & $11.218 \mathrm{aA}-\mathrm{A}$ & $4.159 \mathrm{aB}-\mathrm{B}$ & $11.171 \mathrm{aA}-\mathrm{A}$ \\
\hline P. guenoarum Baio & $4.382 \mathrm{bA}-\mathrm{B}$ & $9.850 \mathrm{aB}-\mathrm{A}$ & $3.973 \mathrm{aA}-\mathrm{B}$ & $12.042 \mathrm{aA}-\mathrm{A}$ \\
\hline P. nicorae 26A & $1.641 \mathrm{cB}-\mathrm{B}$ & $3.443 \mathrm{bA}-\mathrm{A}$ & $2.827 \mathrm{bA}-\mathrm{B}$ & $4.813 \mathrm{bA}-\mathrm{A}$ \\
\hline P. nicorae 26D & $2.438 \mathrm{cA}-\mathrm{B}$ & $5.336 \mathrm{bA}-\mathrm{A}$ & $2.428 \mathrm{bA}-\mathrm{B}$ & $3.520 \mathrm{cB}-\mathrm{A}$ \\
\hline P. nicorae $28 \mathrm{~B}$ & $1.877 \mathrm{cB}-\mathrm{B}$ & $4.606 \mathrm{bA}-\mathrm{A}$ & $2.492 \mathrm{bA}-\mathrm{B}$ & $3.860 \mathrm{cA}-\mathrm{A}$ \\
\hline P. nicorae $28 \mathrm{C}$ & $1.783 \mathrm{cB}-\mathrm{B}$ & $4.074 \mathrm{bA}-\mathrm{A}$ & $2.538 \mathrm{bA}-\mathrm{B}$ & $4.847 \mathrm{bA}-\mathrm{A}$ \\
\hline P. nicorae $28 \mathrm{E}$ & $1.489 \mathrm{cB}-\mathrm{A}$ & $1.804 \mathrm{cB}-\mathrm{A}$ & $2.409 \mathrm{bA}-\mathrm{B}$ & $3.847 \mathrm{cA}-\mathrm{A}$ \\
\hline P. notatum Pensacola & $2.175 \mathrm{cA}-\mathrm{B}$ & $4.899 \mathrm{bA}-\mathrm{A}$ & $2.018 \mathrm{bA}-\mathrm{A}$ & $2.449 \mathrm{~dB}-\mathrm{A}$ \\
\hline \multirow[t]{2}{*}{ Média geral } & 2.663 & 5.654 & 2.856 & 5.819 \\
\hline & \multicolumn{4}{|c|}{ Massa de matéria seca de colmos $\left(\mathrm{kg} \mathrm{ha}^{-1}\right)$} \\
\hline P. guenoarum Azulão & 884bB-B & $4.141 \mathrm{aA}-\mathrm{A}$ & $2.768 \mathrm{aA}-\mathrm{A}$ & $1.490 \mathrm{bB}-\mathrm{B}$ \\
\hline P. guenoarum Baio & $1.608 \mathrm{aB}-\mathrm{B}$ & $4.402 \mathrm{aA}-\mathrm{A}$ & $2.505 \mathrm{aA}-\mathrm{B}$ & $4.416 \mathrm{aA}-\mathrm{A}$ \\
\hline P. nicorae 26A & $1.076 \mathrm{bB}-\mathrm{A}$ & $1.434 \mathrm{cA}-\mathrm{A}$ & $1.765 \mathrm{bA}-\mathrm{A}$ & $991 \mathrm{bA}-\mathrm{B}$ \\
\hline P. nicorae 26D & $1.124 \mathrm{bB}-\mathrm{A}$ & $1.050 \mathrm{cA}-\mathrm{A}$ & $1.642 \mathrm{bA}-\mathrm{A}$ & $738 \mathrm{bA}-\mathrm{B}$ \\
\hline P. nicorae 28B & $1.581 \mathrm{aB}-\mathrm{B}$ & $3.124 \mathrm{bA}-\mathrm{A}$ & $2.155 \mathrm{aA}-\mathrm{A}$ & $1.241 \mathrm{bB}-\mathrm{B}$ \\
\hline P. nicorae $28 \mathrm{C}$ & $1.318 \mathrm{bA}-\mathrm{A}$ & $1.073 \mathrm{cA}-\mathrm{A}$ & $1.523 \mathrm{bA}-\mathrm{A}$ & $1.156 \mathrm{bA}-\mathrm{A}$ \\
\hline P. nicorae $28 \mathrm{E}$ & $1.241 \mathrm{bB}-\mathrm{A}$ & $800 \mathrm{cA}-\mathrm{A}$ & $2.368 \mathrm{aA}-\mathrm{A}$ & $1.024 \mathrm{bA}-\mathrm{B}$ \\
\hline P. notatum Pensacola & $1.211 \mathrm{bA}-\mathrm{A}$ & $536 \mathrm{cA}-\mathrm{A}$ & $1.583 \mathrm{bA}-\mathrm{A}$ & $601 \mathrm{bA}-\mathrm{B}$ \\
\hline Média geral & 1.255 & 3.574 & 2.039 & 1.457 \\
\hline
\end{tabular}


No ano de estabelecimento, os acessos Azulão, Baio, 26A e 28E produziram mais matéria seca total em Augusto Pestana do que em Eldorado do Sul. No segundo ano, no entanto, a produção de MST em Eldorado do Sul, em geral, foi superior.

Os maiores valores médios de MSF e MSC também ocorreram no ano seguinte à implantação (Tabela 2). Ressalta-se que a produção de colmos é importante na sustentação dos afilhos e de folhas; no entanto, sua maior expressão compromete a estrutura do dossel e diminui a relação folha:colmo (Gomide et al., 2007). A seleção para a redução da produção de colmo e aumento da de folha tem sido fortemente preconizada no melhoramento de forrageiras (Pereira et al., 2011), pois a maior qualidade nutricional está na lâmina foliar, estrutura preferida pelo animal em regime de pastejo (Bratti et al., 2009).

Destaca-se que os acessos Azulão e Baio, de P. guenoarum, apresentaram maior estabilidade entre locais e as maiores produções de matéria seca total e de folhas na comparação entre os acessos (Tabela 2). Isso indica a presença de alelos de adaptação, que têm forte interesse para programas de melhoramento, na obtenção de recombinantes superiores.

No teste de médias para a RFC, em Eldorado do Sul, os acessos diferiram, em ambos os anos de cultivo (Tabela3). Osacessos Baio, 26Ae28B não apresentaram diferenças entre os resultados no ano de estabelecimento e no segundo ano. No entanto, os acessos Azulão, 26D, $28 \mathrm{C}$ e $28 \mathrm{E}$ apresentaram aumento em RFC, no segundo

Tabela 3. Relação folha:colmo, índice de colheita e número de afilhos por metro, em acessos do gênero Paspalum, em dois anos de cultivo e em dois municípios do Rio Grande do $\mathrm{Sul}^{(1)}$.

\begin{tabular}{|c|c|c|c|c|}
\hline \multirow[t]{2}{*}{ Genótipo } & \multicolumn{2}{|c|}{ Eldorado do Sul } & \multicolumn{2}{|c|}{ Augusto Pestana } \\
\hline & $2009 / 2010$ & $2010 / 2011$ & $2009 / 2010$ & $2010 / 2011$ \\
\hline & \multicolumn{4}{|c|}{ Relação folha:colmo (MSF/MSC) } \\
\hline P. guenoarum Azulão & $6,37 \mathrm{aA}-\mathrm{A}$ & $2,78 \mathrm{~dB}-\mathrm{B}$ & $1,51 \mathrm{aB}-\mathrm{B}$ & $7,70 \mathrm{aA}-\mathrm{A}$ \\
\hline P. guenoarum Baio & $2,75 \mathrm{bA}-\mathrm{A}$ & $2,22 \mathrm{dA}-\mathrm{A}$ & $1,61 \mathrm{aB}-\mathrm{B}$ & $2,83 \mathrm{bA}-\mathrm{A}$ \\
\hline P. nicorae $26 \mathrm{~A}$ & $1,52 \mathrm{cA}-\mathrm{A}$ & $2,41 \mathrm{~dB}-\mathrm{A}$ & $1,65 \mathrm{aA}-\mathrm{B}$ & $4,87 \mathrm{bA}-\mathrm{A}$ \\
\hline P. nicorae 26D & $2,16 \mathrm{bA}-\mathrm{B}$ & $5,08 \mathrm{bA}-\mathrm{A}$ & $1,55 \mathrm{aA}-\mathrm{B}$ & $4,82 \mathrm{bA}-\mathrm{A}$ \\
\hline P. nicorae $28 \mathrm{~B}$ & $1,19 \mathrm{cA}-\mathrm{A}$ & $1,51 \mathrm{~dB}-\mathrm{A}$ & $1,16 \mathrm{bA}-\mathrm{B}$ & $3,12 \mathrm{bA}-\mathrm{A}$ \\
\hline P. nicorae $28 \mathrm{C}$ & $1,37 \mathrm{cA}-\mathrm{B}$ & $3,83 \mathrm{cA}-\mathrm{A}$ & $1,68 \mathrm{aA}-\mathrm{B}$ & $4,18 \mathrm{bA}-\mathrm{A}$ \\
\hline P. nicorae $28 \mathrm{E}$ & $1,19 \mathrm{cA}-\mathrm{B}$ & $2,26 \mathrm{~dB}-\mathrm{A}$ & $1,03 \mathrm{bA}-\mathrm{B}$ & $3,85 \mathrm{bA}-\mathrm{A}$ \\
\hline P. notatum Pensacola & $1,83 \mathrm{cA}-\mathrm{B}$ & $9,18 \mathrm{aA}-\mathrm{A}$ & $1,27 \mathrm{bA}-\mathrm{B}$ & $4,07 \mathrm{bB}-\mathrm{A}$ \\
\hline \multirow[t]{2}{*}{ Média geral } & 2,30 & 1,58 & 1,43 & 3,99 \\
\hline & \multicolumn{4}{|c|}{ Índice de colheita (MSF/MST) } \\
\hline P. guenoarum Azulão & $0,84 \mathrm{aA}-\mathrm{A}$ & $0,70 \mathrm{cB}-\mathrm{B}$ & $0,51 \mathrm{aB}-\mathrm{B}$ & $0,84 \mathrm{aA}-\mathrm{A}$ \\
\hline P. guenoarum Baio & $0,70 \mathrm{bA}-\mathrm{A}$ & $0,68 \mathrm{cA}-\mathrm{A}$ & $0,53 \mathrm{aB}-\mathrm{B}$ & $0,71 \mathrm{cA}-\mathrm{A}$ \\
\hline P. nicorae $26 \mathrm{~A}$ & $0,42 \mathrm{~dB}-\mathrm{B}$ & $0,70 \mathrm{cB}-\mathrm{A}$ & $0,51 \mathrm{aA}-\mathrm{B}$ & $0,83 \mathrm{aA}-\mathrm{A}$ \\
\hline P. nicorae $26 \mathrm{D}$ & $0,60 \mathrm{cA}-\mathrm{B}$ & $0,82 \mathrm{bA}-\mathrm{A}$ & $0,50 \mathrm{aB}-\mathrm{B}$ & $0,83 \mathrm{aA}-\mathrm{A}$ \\
\hline P. nicorae $28 \mathrm{~B}$ & $0,41 \mathrm{dA}-\mathrm{B}$ & $0,57 \mathrm{~dB}-\mathrm{A}$ & $0,46 \mathrm{bA}-\mathrm{B}$ & $0,76 \mathrm{bA}-\mathrm{A}$ \\
\hline P. nicorae $28 \mathrm{C}$ & $0,48 \mathrm{dA}-\mathrm{B}$ & $0,81 \mathrm{bA}-\mathrm{A}$ & $0,55 \mathrm{aA}-\mathrm{B}$ & $0,81 \mathrm{aA}-\mathrm{A}$ \\
\hline P. nicorae $28 \mathrm{E}$ & $0,41 \mathrm{dA}-\mathrm{B}$ & $0,66 \mathrm{cB}-\mathrm{A}$ & $0,41 \mathrm{bA}-\mathrm{B}$ & $0,80 \mathrm{aA}-\mathrm{A}$ \\
\hline P. notatum Pensacola & $0,55 \mathrm{cA}-\mathrm{B}$ & $0,90 \mathrm{aA}-\mathrm{A}$ & $0,45 \mathrm{bB}-\mathrm{B}$ & $0,80 \mathrm{aB}-\mathrm{A}$ \\
\hline \multirow[t]{2}{*}{ Média geral } & 0,55 & 0,73 & 0,49 & 0,80 \\
\hline & \multicolumn{4}{|c|}{ Número de afilhos por $\mathrm{m}^{2}$} \\
\hline P. guenoarum Azulão & $359 \mathrm{aA}-\mathrm{B}$ & $543 \mathrm{aA}-\mathrm{A}$ & $446 \mathrm{bA}-\mathrm{A}$ & $592 \mathrm{bA}-\mathrm{A}$ \\
\hline P. guenoarum Baio & $388 \mathrm{aA}-\mathrm{B}$ & $553 \mathrm{aA}-\mathrm{A}$ & $480 \mathrm{bA}-\mathrm{A}$ & $552 \mathrm{bA}-\mathrm{A}$ \\
\hline P. nicorae $26 \mathrm{~A}$ & $476 \mathrm{aA}-\mathrm{A}$ & $323 b B-B$ & $595 \mathrm{bA}-\mathrm{B}$ & $972 \mathrm{aA}-\mathrm{A}$ \\
\hline P. nicorae 26D & $398 \mathrm{aB}-\mathrm{B}$ & $580 \mathrm{aB}$ & $631 \mathrm{bA}-\mathrm{B}$ & $904 \mathrm{aA}-\mathrm{A}$ \\
\hline P. nicorae $28 \mathrm{~B}$ & $497 \mathrm{aB}-\mathrm{A}$ & $443 \mathrm{aB}$ & $743 \mathrm{aA}-\mathrm{A}$ & $910 \mathrm{aA}-\mathrm{A}$ \\
\hline P. nicorae $28 \mathrm{C}$ & $481 \mathrm{aA}-\mathrm{A}$ & $494 \mathrm{aB}$ & $570 \mathrm{bA}-\mathrm{B}$ & $879 \mathrm{aA}-\mathrm{A}$ \\
\hline P. nicorae $28 \mathrm{E}$ & $544 \mathrm{aA}-\mathrm{A}$ & $260 \mathrm{bB}-\mathrm{B}$ & $571 \mathrm{bA}-\mathrm{B}$ & $950 \mathrm{aA}-\mathrm{A}$ \\
\hline P. notatum Pensacola & $541 \mathrm{aB}-\mathrm{A}$ & $213 \mathrm{bB}-\mathrm{B}$ & $876 \mathrm{aA}-\mathrm{A}$ & $500 \mathrm{bA}-\mathrm{B}$ \\
\hline Média geral & 461 & 426 & 614 & 782 \\
\hline
\end{tabular}


ano de avaliação. Para a cultivar Pensacola, observouse alto acréscimo na produção de folhas do primeiro para o segundo ano, quando comparado aos demais acessos. No ano de estabelecimento (2009/2010), o acesso Azulão apresentou desempenho superior aos demais, na produção de folhas.

Em Augusto Pestana, o segundo ano possibilitou maior média de RFC em todos os genótipos (Tabela 3). No primeiro ano, os maiores valores da RFC foram obtidos pelos acessos Azulão, Baio, 26A, 26D e 28C. No ano seguinte, apenas o acesso Azulão destacou-se em relação aos demais.

Na comparação entre os locais, em 2009/2010 foram observadas semelhanças para a maioria dos acessos; porém, Azulão e Baio, em Eldorado do Sul, apresentaram o melhor desempenho. No ano seguinte, os acessos Azulão, 28B e 28C, em Augusto Pestana, tiveram os melhores desempenhos. Bratti et al. (2009) consideram a relação RFC um dos parâmetros fundamentais na análise da seleção dos animais em pastejo.

Em relação ao índice de colheita em Eldorado do Sul, os genótipos também evidenciaram melhores resultados no segundo ano, exceto os acessos Azulão e Baio. Em Augusto Pestana, os genótipos mostraram maiores valores de IC no segundo ano, sobretudo para o acesso Azulão. Na comparação entre locais, grande parte dos acessos mostraram superioridade em IC em Augusto Pestana, no segundo ano. O IC expressa a fração de matéria seca não exportada das folhas para outras partes da planta (Benincasa, 1988) e constitui fator importante no melhoramento de forrageiras.

Em Augusto Pestana ocorreram os maiores valores de NAF, na grande maioria dos genótipos (Tabela 3).
No entanto, verificou-se reduzida variabilidade genética neste caráter, com baixa variação entre os genótipos. Assim, em 2009/2010, não foram constatadas diferenças entre os acessos e, no ano seguinte, foram observadas apenas duas classes fenotípicas para discriminação dos genótipos, tanto em Eldorado do Sul quanto em Augusto Pestana. A seleção pelo caráter afilhamento ainda é bastante questionada no melhoramento de plantas (Kuraparthy et al., 2007).

Quanto à contribuição relativa dos caracteres ligados ao potencial forrageiro para a variabilidade total observada (Tabela 4), foram observadas diferenças na capacidade de expressão dessas variáveis em Eldorado do Sul e Augusto Pestana, inclusive com efeito cumulativo destes locais sobre a variabilidade total. Alves et al. (2003) relataram a importância da determinação da contribuição relativa dos caracteres para possibilitar a redução no número de avaliações necessárias para determinação da variabilidade genética, já que apenas caracteres que efetivamente contribuem para essa variabilidade são mensurados. Os anos de avaliação influenciaram a variabilidade observada. Em Eldorado do Sul, a MSF e o IC foram os caracteres que mais contribuíram para a variação, com valores de 68,65 e $23,13 \%$, respectivamente, enquanto os demais caracteres tiveram reduzida participação. Em Augusto Pestana, embora a MSF tenha apresentado efetiva expressão nos acessos testados, a maior participação foi a do caráter MST, com mais de $52 \%$ de contribuição sobre a variabilidade genética total entre os acessos.

No ano de estabelecimento, na média dos dois locais, a maior proporção da variabilidade genética foi devida a MSF e a MST, com valores de 53,33 e 25,7\%,

Tabela 4. Contribuição relativa para a variabilidade observada nos caracteres ligados ao potencial forrageiro de acessos do gênero Paspalum.

\begin{tabular}{|c|c|c|c|c|c|c|c|}
\hline Ambiente & Análise conjunta & MST & MSF & MSC & RFC & $\mathrm{IC}$ & NAF \\
\hline Eldorado do Sul & \multirow{2}{*}{ Anos } & 2,61 & 488,18 & 28,3 & 22,3 & 164,50 & 4,52 \\
\hline Contribuição (\%) & & 0,36 & 68,65 & 3,98 & 3,22 & 23,13 & 0,63 \\
\hline Augusto Pestana & \multirow{2}{*}{ Anos } & 3.350 & 2.456 & 433 & 11,64 & 92,17 & 7,40 \\
\hline Contribuição (\%) & & 52,74 & 38,67 & 6,82 & 0,18 & 1,45 & 0,11 \\
\hline Primeiro ano & \multirow{2}{*}{ Locais } & 473,89 & 983,49 & 120,66 & 6,45 & 180,99 & 78,3 \\
\hline Contribuição (\%) & & 25,70 & 53,33 & 6,54 & 0,35 & 9,81 & 4,24 \\
\hline Segundo ano & \multirow{2}{*}{ Locais } & 1.358 & 2.046 & 55,75 & 40,87 & 219,80 & 68,70 \\
\hline Contribuição (\%) & & 35,84 & 53,98 & 1,47 & 1,07 & 5,79 & 1,81 \\
\hline Geral & \multirow{2}{*}{ Anos + locais } & 435,12 & 211,75 & 31,01 & 14,32 & 74,32 & 12,90 \\
\hline Contribuição (\%) & & 55,80 & 27,16 & 3,97 & 1,85 & 9,35 & 1,66 \\
\hline
\end{tabular}

MST, matéria seca total; MSF, matéria seca de folha; MSC, matéria seca de colmo; RFC, relação folha:colmo (MSF/MSC); IC, índice de colheita (MSF/ MST); NAF, número de afilhos. 
respectivamente (Tabela 4). No segundo ano, nos dois locais, foram observadas contribuições relativas de 35,84 e 53,98\%, para MST e MSF, respectivamente. Contudo, na contribuição geral, que inclui o efeito cumulativo dos anos e dos locais, confirmou-se a maior efetividade dos caracteres MST $(55,8 \%)$ e MSF (27,16\%), para quantificar a variabilidade entre os acessos. Caracteres que contribuem pouco para a divergência genética não devem ser utilizados na seleção por diminuir a eficiência do processo (Alves, et al., 2003; Moreira et al., 2005). Pereira et al. (2011) também obtiveram maior contribuição de MST e MSF para avaliação da variabilidade genética no gênero Paspalum.

A análise de agrupamento pelo método de Tocher (Tabela 5) possibilitou a distribuição dos oito acessos avaliados em quatro grupos distintos, em Eldorado do Sul. Em Augusto Pestana, obteve-se a formação de apenas dois grupos, na análise conjunta dos dois anos de avaliação. O maior número de grupos formados em Eldorado do Sul indica que esse ambiente é mais favorável à seleção, pois permite a expressão de maior variabilidade entre os genótipos. Entretanto, em Augusto Pestana, o ambiente se mostrou mais favorável para a expressão do potencial genético, na maioria dos acessos estudados (Tabelas 2 e 3 ).

Ao se considerar os resultados obtidos em Eldorado do Sul, os acessos apomíticos 28C, 26D e 28B de

Tabela 5. Discriminação da variabilidade genética de acessos do gênero Paspalum por meio da análise de agrupamento de Tocher $^{(1)}$.

\begin{tabular}{lcc}
\hline Ambientes & Grupos & Agrupamento \\
\hline $\begin{array}{l}\text { Eldorado do Sul } \\
\text { (análise conjunta de anos) }\end{array}$ & I & 26A, 28E, 28C \\
& III & 26D, Pensacola \\
\hline Augusto Pestana & IV & Azulão, Baio \\
(análise conjunta de anos) & I & 26A, 28E, 28C, 26D, 28B, \\
\hline 2009/2010 & II & Pensacola \\
(análise conjunta de locais) & I & 26A, 28E, 28C, 26D, 28B, \\
& & Pensacola \\
\hline II & Azulão, Baio \\
(análise conjunta de locais) & I & 26A, 28E, 28C, 26D, 28B \\
& II & Azulão, Baio \\
\hline Geral & II & Pensacola \\
(análise conjunta de locais e anos) & I & 26A, 28E, 28C, 26D, 28B, \\
& II & Pensacola \\
\hline
\end{tabular}

P. nicorae, e o acesso Azulão de P. guenoarum podem ser utilizados como genitores masculinos na combinação com o genitor sexual feminino. No entanto, com base nos resultados encontrados em Augusto Pestana, apenas o acesso $28 \mathrm{C}$ de $P$. nicorae e o acesso Azulão de $P$. guenoarum devem ser indicados para cruzamentos com o genitor sexual.

\section{Conclusões}

1. Os acessos avaliados apresentam variabilidade genética em caracteres de interesse forrageiro, bem como desempenho variável de acordo com o local e o ano de cultivo.

2. A produção de matéria seca total e de folhas são os caracteres que mais contribuem para a detecção da variabilidade genética observada, independentemente do ano de avaliação.

\section{Referências}

ACUÑA, C.A.; BLOUNT, A.R.; QUESENBERRY, K.H.; KENWORTHY, K.E.; HANNA, W.W. Bahiagrass tetraploid germplasm: reproductive and agronomic characterization of segregating progeny. Crop Science, v.49, p.581-588, 2009.

ADAMOWSKI,E.deV.;PAGLIARINI,M.S.;MENDES-BONATO, A.B.; BATISTA, L.A.R.; VALLS, J.F.M. Chromosome numbers and meiotic behavior of some Paspalum accessions. Genetic and Molecular Biology, v.28, p.773-780, 2005.

AGUILERA, P.M.; SARTOR, M.E.; GALDEANO, F.; ESPINOZA, F.; QUARIN, C.L. Interspecific tetraploid hybrids between two forage grass species: sexual Paspalum plicatulum and apomictic $P$. guenoarum. Crop Science, v.51, p.1544-1550, 2011.

ALVES, R.M.; GARCIA A.A.F.; CRUZ, E.D.; FIGUEIRA, A. Seleção de descritores botânico-agronômicos para caracterização de germoplasma de cupuaçuzeiro. Pesquisa Agropecuária Brasileira, v.38, p.807-818, 2003.

ASSIS, G.M.L. de; VALENTIM, J.F.; CARNEIRO JÚNIOR, J.M.; AZEVEDO, J.M.A. de; FERREIRA, A.S. Seleção de genótipos de amendoim forrageiro para cobertura do solo e produção de biomassa aérea no período de estabelecimento utilizando-se metodologia de modelos mistos. Revista Brasileira de Zootecnia, v.37, p.1905-1911, 2008.

BATISTA, L.A.R.; GODOY, R. Caracterização preliminar e seleção de germoplasma de gênero Paspalum para produção de forragem. Revista Brasileira de Zootecnia, v.29, p.23-32, 2000.

BENINCASA, M.M.P. Análise de crescimento de plantas: noções básicas. Jaboticabal: Unesp, 1988. 42p.

BRATTI, L.F.S.; DITTRICH, J.R.; BARROS, C.S. de; SILVA, C.J.A. da; MONTEIRO, A.L.G.; ROCHA, C. da; ROCHA, F.M.P. da. Comportamento ingestivo de caprinos em pastagem de azevém 
e aveia-preta em cultivo puro e consorciado. Ciência Animal Brasileira, v.10, p.397-405, 2009.

CRUZ, C.D. Programa GENES: aplicativo computacional em genética e estatística. Viçosa: UFV, 2007. 442p.

GOMIDE, C.A. de M.; GOMIDE, J.A.; ALEXANDRINO, E. Características estruturais e produção de forragem em pastos de capim-mombaça submetidos a períodos de descanso. Pesquisa Agropecuária Brasileira, v.42, p.1487-1494, 2007.

KURAPARTHY, V.; SOOD, S.; DHALIWAL, H.S.; CHHUNEJA, P.; GILL, B.S. Identification and mapping of a tiller inhibition gene (tin3) in wheat. Theoretical and Applied Genetics, v.114, p.285-294, 2007.

MANUAL de adubação e de calagem para os Estados do Rio Grande do Sul e Santa Catarina. 10.ed. Porto Alegre: Sociedade Brasileira de Ciência do Solo - Núcleo Regional Sul - Comissão de Química e Fertilidade do Solo, 2004. 400p.

MOREIRA, G.R.; SILVA, D.J.H. da; PICANÇO, M.C.; PETERNELLI, L.A.; CALIMAN, F.R.B. Divergência genética entre acessos de tomateiro infestados por diferentes populações da traça-do-tomateiro. Horticultura Brasileira, v.23, p.887-892, 2005.

PEREIRA, E.A.; DALL'AGNOL, M.; NABINGER, C.; HUBER, K.G.C.; MONTARDO, D.P.; GENRO, T.C.M.Produção agronômica de uma coleção de acessos de Paspalum nicorae Parodi. Revista Brasileira de Zootecnia, v.40, p.498-508, 2011.
QUARÍN, C.L.; NORRMANN, G.A. Interspecific hybrids between five Paspalum species. Botanical Gazette, v.151, p.366-369, 1990.

REIS, C.A. de O. dos; DALL'AGNOL, M.; NABINGER, C.; SCHIFINO-WITTMANN, M.T. Morphological variation in Paspalum nicorae Parodi accessions, a promising forage. Scientia Agricola, v.67, p.143-150, 2010.

RODRIGUES, J.C.M.; CABRAL, G.B.; DUSI, D.M.A.; MELLO, L.V.; RIGDEN, D.; CARNEIRO, V.T.C. Identification of differentially expressed cDNA sequences in ovaries of sexual and apomictic plants of Brachiaria brizantha. Plant Molecular Biology, v.23, p.745-757, 2003.

SARTOR, M.E.; QUARIN, C.L.; ESPINOZA, F. Mode of reproduction of colchicine-induced Paspalum plicatulum tetraploids. Crop Science, v.49, p.1270-1276, 2009.

SAWASATO, J.T. Caracterização agronômica e molecular de Paspalum urvillei Steudel. 2007. 109p. Dissertação (Mestrado) Universidade Federal do Rio Grande do Sul, Porto Alegre.

SINGH, D. The relative importance of characters affecting genetic divergence. The Indian Journal of Genetics and Plant Breeding, v.41, p.237-245, 1981.

STRAPASSON, E.; VENCOVSKY, R.; BATISTA, L.A.R. Seleção de descritores na caracterização de germoplasma de Paspalum sp. por meio de componentes principais. Revista Brasileira de Zootecnia, v.29, p.373-381, 2000.

Recebido em 30 de abril de 2012 e aprovado em 26 de setembro de 2012 